\title{
Hermite-Hadamard Type Integral Inequalities for Functions Whose Second-Order Mixed Derivatives Are Coordinated $(s, m)-P$-Convex
}

\author{
Yu-Mei Bai, ${ }^{1}$ Shan-He Wu $\left(D,{ }^{2}\right.$ and Ying Wu ${ }^{1}$ \\ ${ }^{1}$ College of Mathematics, Inner Mongolia University for Nationalities, Tongliao, \\ Inner Mongolia Autonomous Region 028043, China \\ ${ }^{2}$ Department of Mathematics, Longyan University, Longyan, Fujian 364012, China \\ Correspondence should be addressed to Shan-He Wu; shanhewu@gmail.com
}

Received 29 October 2017; Accepted 11 January 2018; Published 6 February 2018

Academic Editor: Adrian Petrusel

Copyright (C) 2018 Yu-Mei Bai et al. This is an open access article distributed under the Creative Commons Attribution License, which permits unrestricted use, distribution, and reproduction in any medium, provided the original work is properly cited.

\begin{abstract}
We establish some new Hermite-Hadamard type integral inequalities for functions whose second-order mixed derivatives are coordinated $(s, m)$ - $P$-convex. An expression form of Hermite-Hadamard type integral inequalities via the beta function and the hypergeometric function is also presented. Our results provide a significant complement to the work of Wu et al. involving the Hermite-Hadamard type inequalities for coordinated $(s, m)-P$-convex functions in an earlier article.
\end{abstract}

\section{Introduction}

Let $f: I \rightarrow \mathbb{R}$ be a convex mapping. Then for any $a, b \in I$ with $a<b$, we have the following double inequality:

$$
f\left(\frac{a+b}{2}\right) \leq \frac{1}{b-a} \int_{a}^{b} f(x) d x \leq \frac{f(a)+f(b)}{2} .
$$

This celebrated inequality is known in the literature as the Hermite-Hadamard inequality. As we all know, some of the classical inequalities for means can be derived from (1) for appropriate particular selections of the mapping $f$. Indeed, Hermite-Hadamard's inequality (1) has already found many applications in mathematical analysis and optimization (see, for example, [1-9]).

In recent years, the applications of various properties of extended convex functions in establishing and improving Hermite-Hadamard type inequalities have attracted the attention of many researchers (see [10-15] and references cited therein).

In [16], Wu et al. established some Hermite-Hadamard type inequalities under the assumption that the function $f$ is a coordinated $(s, m)$-P-convex function. Motivated by the ideas of work [16], in this paper we study Hermite-Hadamard type inequalities related to the convexity of second-order mixed derivatives of $f$. More precisely, we focus on establishing some new Hermite-Hadamard type inequalities for functions whose second-order mixed derivatives are coordinated $(s, m)-P$-convex. For convenience of our discussions in subsequent sections, we begin with recalling some relevant definitions.

Definition 1. A function $f: I \subseteq \mathbb{R} \rightarrow \mathbb{R}$ is said to be convex function if

$$
f(t x+(1-t) y) \leq t f(x)+(1-t) f(y)
$$

holds for all $x, y \in I$ and $t \in[0,1]$.

Definition 2 (see [5]). We say that a map $f: I \subseteq \mathbb{R} \rightarrow \mathbb{R}$ belongs to the class $P(I)$ if it is nonnegative and for all $x, y \in I$ and $t \in[0,1]$ satisfies the following inequality:

$$
f(t x+(1-t) y) \leq f(x)+f(y) .
$$

In [17], the concept of $m$-convex functions was introduced as follows. 
Definition 3 (see [17]). For $f:[0, b] \subseteq \mathbb{R}_{0}=[0,+\infty) \rightarrow \mathbb{R}$ and $m \in(0,1]$, if

$$
f(t x+m(1-t) y) \leq t f(x)+m(1-t) f(y)
$$

is valid for all $x, y \in[0, b]$ and $t \in[0,1]$, then we say that $f$ is a $m$-convex function on $[0, b]$.

In [18], the concept of $s$-convex functions was presented as follows.

Definition 4 (see [18]). Let $s \in(0,1]$. A function $f: I \subseteq \mathbb{R} \rightarrow$ $\mathbb{R}$ is said to be $s$-convex (in the second sense) if

$$
f(t x+(1-t) y) \leq t^{s} f(x)+(1-t)^{s} f(y)
$$

holds for all $x, y \in I$ and $t \in[0,1]$.

Definition 5 (see [19]). For $(s, m) \in(0,1] \times(0,1]$, a function $f:[0, b] \rightarrow \mathbb{R}$ is said to be $(s, m)$-convex if

$$
f(t x+m(1-t) y) \leq t^{s} f(x)+m(1-t)^{s} f(y)
$$

holds for all $x, y \in I$ and $t \in[0,1]$.

Definition 6 (see [20]). For some $s \in[-1,1]$, a function $f$ : $I \subseteq \mathbb{R} \rightarrow \mathbb{R}$ is said to be extended $s$-convex if

$$
f(t x+(1-t) y) \leq t^{s} f(x)+(1-t)^{s} f(y)
$$

is valid for all $x, y \in I$ and $t \in(0,1)$.

Dragomir [21] and Dragomir and Pearce [22] considered the convexity of a function on the coordinates and put forward the following definition.

Definition 7 (see $[21,22])$. A function $f: \Delta=[a, b] \times[c, d] \subseteq$ $\mathbb{R}^{2} \rightarrow \mathbb{R}$ is said to be convex on the coordinates on $\Delta$ with $a<b$ and $c<d$ if the partial functions

$$
\begin{aligned}
f_{y}:[a, b] & \longrightarrow \mathbb{R}, \\
f_{y}(u) & =f(u, y), \\
f_{x}:[c, d] & \longrightarrow \mathbb{R}, \\
f_{x}(v) & =f(x, v),
\end{aligned}
$$

are convex for all $x \in(a, b)$ and $y \in(c, d)$.

It should be noted that a formal definition for coordinated convex functions is stated as follows.

Definition 8 (see $[21,22])$. A function $f: \Delta=[a, b] \times[c, d] \subseteq$ $\mathbb{R}^{2} \rightarrow \mathbb{R}$ is said to be convex on the coordinates on $\Delta$ with $a<b$ and $c<d$ if the partial function

$$
\begin{aligned}
f(t x & +(1-t) z, \lambda y+(1-\lambda) w) \\
\leq & t \lambda f(x, y)+t(1-\lambda) f(x, w)+(1-t) \lambda f(z, y) \\
& +(1-t)(1-\lambda) f(z, w)
\end{aligned}
$$

holds for all $t, \lambda \in[0,1],(x, y),(z, w) \in \Delta$.
Definition 9 (see [16]). For some $m \in(0,1]$ and $s \in[-1,1]$, a function $f:[0, b] \times[c, d] \rightarrow \mathbb{R}$ is said to be coordinated $(s, m)$-P-convex on $[0, b] \times[c, d]$ with $0<b$ and $c<d$, if

$$
\begin{aligned}
f(t x & +m(1-t) z, \lambda y+(1-\lambda) w) \\
\leq & t^{s}[f(x, y)+f(x, w)] \\
& +m(1-t)^{s}[f(z, y)+f(z, w)]
\end{aligned}
$$

holds for all $t \in(0,1), \lambda \in[0,1]$ and $(x, y),(z, w) \in[0, b] \times$ $[c, d]$.

Dragomir [21] and Dragomir and Pearce [22] established the following result.

Theorem 10 (see $[21,22])$. Let $f: \Delta=[a, b] \times[c, d]$ be convex on the coordinates on $\Delta=[a, b] \times[c, d]$ with $a<b$ and $c<d$. Then, one has the inequalities:

$$
\begin{aligned}
& f\left(\frac{a+b}{2}, \frac{c+d}{2}\right) \leq \frac{1}{2}\left[\frac{1}{b-a} \int_{a}^{b} f\left(x, \frac{c+d}{2}\right) d x\right. \\
& \left.+\frac{1}{d-c} \int_{c}^{d} f\left(\frac{a+b}{2}, y\right) d y\right] \leq \frac{1}{(b-a)(d-c)} \\
& \quad \cdot \int_{a}^{b} \int_{c}^{d} f(x, y) d y d x \\
& \quad \leq \frac{1}{4}\left[\frac{1}{b-a}\left(\int_{a}^{b} f(x, c) d x+\int_{a}^{b} f(x, d) d x\right)\right. \\
& \left.+\frac{1}{d-c}\left(\int_{c}^{d} f(a, y) d y+\int_{c}^{d} f(b, y) d y\right)\right] \\
& \quad \leq \frac{f(a, c)+f(b, c)+f(a, d)+f(b, d)}{4} .
\end{aligned}
$$

In this paper, we shall establish some new integral inequalities of Hermite-Hadamard type for coordinated $(s, m)$-P-convex functions.

\section{Lemma}

Lemma 11 (see [23]). If $f: \Delta=[a, b] \times[c, d] \subseteq \mathbb{R}^{2} \rightarrow \mathbb{R}$ has partial derivatives and $\partial^{2} f / \partial x \partial y \in L_{1}(\Delta)$ with $a<b$ and $c<d$, then

$$
\begin{aligned}
& P(a, b, c, d) \triangleq \frac{1}{(b-a)(d-c)} \int_{a}^{b} \int_{c}^{d} f(x, y) d y d x \\
& +f\left(\frac{a+b}{2}, \frac{c+d}{2}\right)-\frac{1}{b-a} \int_{a}^{b} f\left(x, \frac{c+d}{2}\right) d x
\end{aligned}
$$




$$
\begin{aligned}
& -\frac{1}{d-c} \int_{c}^{d} f\left(\frac{a+b}{2}, y\right) d y=(b-a)(d-c) \quad \text { (1) if } s \in(-1,1] \text {, we have } \\
& \cdot \int_{0}^{1} \int_{0}^{1} K(t, \lambda) \frac{\partial^{2}}{\partial x \partial y} \quad|P(a, b, c, d)| \leq \frac{(b-a)(d-c)}{2^{3} \times 2^{(s+1) / q}}\left\{\left[\frac{1}{s+2} \Delta_{1}(q)\right.\right. \\
& \cdot f(t a+(1-t) b, \lambda c+(1-\lambda) d) d t d \lambda \\
& \left.+m \frac{2^{s+2}-s-3}{(s+1)(s+2)} \Delta_{2}(m, q)\right]^{1 / q}
\end{aligned}
$$

where

$$
\begin{aligned}
& K(t, \lambda) \\
& \quad= \begin{cases}t \lambda, & (t, \lambda) \in\left[0, \frac{1}{2}\right] \times\left[0, \frac{1}{2}\right], \\
t(\lambda-1), & (t, \lambda) \in\left[0, \frac{1}{2}\right] \times\left(\frac{1}{2}, 1\right], \\
(t-1) \lambda, & (t, \lambda) \in\left(\frac{1}{2}, 1\right] \times\left[0, \frac{1}{2}\right], \\
(t-1)(\lambda-1), & (t, \lambda) \in\left(\frac{1}{2}, 1\right] \times\left(\frac{1}{2}, 1\right] .\end{cases}
\end{aligned}
$$$$
\left.+\left[\frac{2^{s+2}-s-3}{(s+1)(s+2)} \Delta_{1}(q)+\frac{m}{s+2} \Delta_{2}(m, q)\right]^{1 / q}\right\},
$$

\section{Main Results}

In this section, we establish some Hermite-Hadamard type integral inequalities for functions whose second-order mixed derivatives are coordinated $(s, m)-P$-convex on the plane $\mathbb{R}_{0} \times$ $\mathbb{R}$.

Theorem 12. Suppose that the function $f: \mathbb{R}_{0} \times \mathbb{R} \rightarrow$ $\mathbb{R}$ has continuous partial derivatives of the second-order and $\partial^{2} f / \partial x \partial y \in L_{1}\left(\left[0, b^{*} / m\right] \times[c, d]\right)$ with $0 \leq a<b \leq b^{*}$, $c<d$, for some $m \in(0,1]$ and $s \in[-1,1]$. If $\left|\partial^{2} f / \partial x \partial y\right|^{q}$ is coordinated $(s, m)$-P-convex functions on $\left[0, b^{*} / m\right] \times[c, d]$ for $q \geq 1$, then where

(2) if $s=-1$, we have

$$
\begin{aligned}
& |P(a, b, c, d)| \leq \frac{(b-a)(d-c)}{8} \\
& \quad \times\left\{\left[\Delta_{1}(q)+m(2 \ln 2-1) \Delta_{2}(m, q)\right]^{1 / q}\right. \\
& \left.\quad+\left[(2 \ln 2-1) \Delta_{1}(q)+m \Delta_{2}(m, q)\right]^{1 / q}\right\},
\end{aligned}
$$

$$
\begin{gathered}
\Delta_{1}(q)=\left|\frac{\partial^{2} f(a, c)}{\partial x \partial y}\right|^{q}+\left|\frac{\partial^{2} f(a, d)}{\partial x \partial y}\right|^{q}, \\
\Delta_{2}(m, q)=\left|\frac{\partial^{2} f(b / m, c)}{\partial x \partial y}\right|^{q}+\left|\frac{\partial^{2} f(b / m, d)}{\partial x \partial y}\right|^{q} .
\end{gathered}
$$

Proof. By Lemma 11 and Hölder's integral inequality, we have

$$
\begin{aligned}
& |P(a, b, c, d)| \leq(b-a)(d-c)\left(\int_{0}^{1} \int_{0}^{1}|K(t, \lambda)| d t d \lambda\right)^{1-1 / q} \\
& \times\left\{\left[\int_{0}^{1 / 2} \int_{0}^{1 / 2} t \lambda\left|\frac{\partial^{2}}{\partial x \partial y} f(t a+(1-t) b, \lambda c+(1-\lambda) d)\right|^{q} d t d \lambda\right]^{1 / q}\right. \\
& +\left[\int_{1 / 2}^{1} \int_{0}^{1 / 2} t(1-\lambda)\left|\frac{\partial^{2}}{\partial x \partial y} f(t a+(1-t) b, \lambda c+(1-\lambda) d)\right|^{q} d t d \lambda\right]^{1 / q} \\
& +\left[\int_{0}^{1 / 2} \int_{1 / 2}^{1}(1-t) \lambda\left|\frac{\partial^{2}}{\partial x \partial y} f(t a+(1-t) b, \lambda c+(1-\lambda) d)\right|^{q} d t d \lambda\right]^{1 / q} \\
& \left.+\left[\int_{1 / 2}^{1} \int_{1 / 2}^{1}(1-t)(1-\lambda)\left|\frac{\partial^{2}}{\partial x \partial y} f(t a+(1-t) b, \lambda c+(1-\lambda) d)\right|^{q} d t d \lambda\right]^{1 / q}\right\} .
\end{aligned}
$$


A straightforward computation gives

$$
\begin{aligned}
\int_{0}^{1 / 2} \lambda d \lambda & =\int_{1 / 2}^{1}(1-\lambda) d \lambda=\frac{1}{8}, \\
\int_{0}^{1 / 2} t^{s+1} d t & =\int_{1 / 2}^{1}(1-t)^{s+1} d t \\
& =\frac{1}{2^{s+5}(s+2)}, \quad \text { for } s \in(-1,1], \\
\int_{0}^{1 / 2} t(1-t)^{s} d t & =\int_{1 / 2}^{1}(1-t) t^{s} d t \\
& =\frac{2^{s+2}-s-3}{2^{s+5}(s+1)(s+2)}, \\
\int_{0}^{1 / 2} t^{-1+1} d t & =\int_{1 / 2}^{1}(1-t)^{-1+1} d t=\frac{1}{2}, \\
\int_{0}^{1} t(1-t)^{-1} d t & =\int_{1 / 2}^{1}(1-t) t^{-1} d t=\ln 2-\frac{1}{2}, \\
\int_{0}^{1}|K(t, \lambda)| d t d \lambda & =\frac{1}{16} .
\end{aligned}
$$

Now, by using the coordinated $(s, m)$-P-convexity of $\left|\partial^{2} f / \partial x \partial y\right|^{q}$, it follows that if $-1<s \leq 1$, we have

$$
\begin{aligned}
& \int_{0}^{1 / 2} \int_{0}^{1 / 2} t \lambda \mid \frac{\partial^{2}}{\partial x \partial y} \\
& \left.\cdot f(t a+(1-t) b, \lambda c+(1-\lambda) d)\right|^{q} d t d \lambda \\
& \quad \leq \int_{0}^{1 / 2} \int_{0}^{1 / 2} t \lambda\left[t^{s} \Delta_{1}(q)+m(1-t)^{s}\right. \\
& \left.\cdot \Delta_{2}(m, q)\right] d t d \lambda=\frac{1}{2^{s+5}(s+2)} \Delta_{1}(q) \\
& +\frac{m\left(2^{s+2}-s-3\right)}{2^{s+5}(s+1)(s+2)} \Delta_{2}(m, q),
\end{aligned}
$$

and if $s=-1$, we have

$$
\begin{aligned}
& \int_{0}^{1 / 2} \int_{0}^{1 / 2} t \lambda\left|\frac{\partial^{2}}{\partial x \partial y} f(t a+(1-t) b, \lambda c+(1-\lambda) d)\right|^{q} d t d \lambda \\
& \quad \leq \int_{0}^{1 / 2} \int_{0}^{1 / 2} t \lambda\left[t^{-1} \Delta_{1}(q)+m(1-t)^{-1} \Delta_{2}(m, q)\right] d t d \lambda \\
& \quad=\frac{1}{16}\left[\Delta_{1}(q)+m(2 \ln 2-1) \Delta_{2}(m, q)\right] .
\end{aligned}
$$

By a similar argument, we obtain

$$
\begin{aligned}
& \int_{1 / 2}^{1} \int_{0}^{1 / 2} t(1-\lambda) \\
& \quad \cdot\left|\frac{\partial^{2}}{\partial x \partial y} f(t a+(1-t) b, \lambda c+(1-\lambda) d)\right|^{q} d t d \lambda \\
& \quad \leq \frac{1}{2^{s+5}} \\
& \quad \times \begin{cases}\frac{1}{s+2} \Delta_{1}(q)+m \frac{2^{s+2}-s-3}{(s+1)(s+2)} \Delta_{2}(m, q), & -1<s \leq 1, \\
\Delta_{1}(q)+m(2 \ln 2-1) \Delta_{2}(m, q), & s=-1,\end{cases}
\end{aligned}
$$

$$
\begin{aligned}
& \int_{0}^{1 / 2} \int_{1 / 2}^{1}(1-t) \\
& \cdot \lambda\left|\frac{\partial^{2}}{\partial x \partial y} f(t a+(1-t) b, \lambda c+(1-\lambda) d)\right|^{q} d t d \lambda \\
& \leq \frac{1}{2^{s+5}} \\
& \times \begin{cases}\frac{2^{s+2}-s-3}{(s+1)(s+2)} \Delta_{1}(q)+\frac{m}{s+2} \Delta_{2}(m, q), & -1<s \leq 1, \\
(2 \ln 2-1) \Delta_{1}(q)+m \Delta_{2}(m, q), & s=-1,\end{cases} \\
& \int_{1 / 2}^{1} \int_{1 / 2}^{1}(1-t)(1-\lambda) \\
& \left|\frac{\partial^{2}}{\partial x \partial y} f(t a+(1-t) b, \lambda c+(1-\lambda) d)\right|^{q} d t d \lambda \\
& \leq \frac{1}{2^{s+5}} \\
& \times \begin{cases}\frac{2^{s+2}-s-3}{(s+1)(s+2)} \Delta_{1}(q)+\frac{m}{s+2} \Delta_{2}(m, q), & -1<s \leq 1, \\
(2 \ln 2-1) \Delta_{1}(q)+m \Delta_{2}(m, q), & s=-1 .\end{cases}
\end{aligned}
$$

Applying (18) and inequalities (19)-(21) into inequality (17), we get (14) and (15). This completes the proof of Theorem 12.

Corollary 13. Under the assumptions of Theorem 12, if $q=1$, then

(1) if $s \in(-1,1]$, then

$|P(a, b, c, d)|$

$$
\leq \frac{(b-a)(d-c)\left(2^{s+1}-1\right)}{2^{s+3}(s+1)(s+2)}\left[\Delta_{1}(1)+m \Delta_{2}(m, 1)\right],
$$

(2) if $s=-1$, then

$$
\begin{aligned}
& |P(a, b, c, d)| \\
& \quad \leq \frac{(b-a)(d-c) \ln 2}{4}\left[\Delta_{1}(1)+m \Delta_{2}(m, 1)\right] .
\end{aligned}
$$

Corollary 14. Under the assumptions of Theorem 12, if $q=$ $m=1$, then

(1) if $s \in(-1,1]$, then

$$
\begin{aligned}
& |P(a, b, c, d)| \leq \frac{(b-a)(d-c)\left(2^{s+1}-1\right)}{2^{s+3}(s+1)(s+2)} \\
& \quad \times\left[\left|\frac{\partial^{2} f(a, c)}{\partial x \partial y}\right|+\left|\frac{\partial^{2} f(a, d)}{\partial x \partial y}\right|+\left|\frac{\partial^{2} f(b, c)}{\partial x \partial y}\right|\right. \\
& \left.\quad+\left|\frac{\partial^{2} f(b, d)}{\partial x \partial y}\right|\right],
\end{aligned}
$$

(2) if $s=-1$, then

$$
\begin{gathered}
|P(a, b, c, d)| \leq \frac{(b-a)(d-c) \ln 2}{4} \times\left[\left|\frac{\partial^{2} f(a, c)}{\partial x \partial y}\right|\right. \\
\left.+\left|\frac{\partial^{2} f(a, d)}{\partial x \partial y}\right|+\left|\frac{\partial^{2} f(b, c)}{\partial x \partial y}\right|+\left|\frac{\partial^{2} f(b, d)}{\partial x \partial y}\right|\right] .
\end{gathered}
$$


Furthermore, if $q=m=1, s=0$, then

$$
\begin{array}{r}
|P(a, b, c, d)| \leq \frac{(b-a)(d-c)}{16} \times\left[\left|\frac{\partial^{2} f(a, c)}{\partial x \partial y}\right|\right. \\
\left.+\left|\frac{\partial^{2} f(a, d)}{\partial x \partial y}\right|+\left|\frac{\partial^{2} f(b, c)}{\partial x \partial y}\right|+\left|\frac{\partial^{2} f(b, d)}{\partial x \partial y}\right|\right] .
\end{array}
$$

Theorem 15. Suppose that the function $f: \mathbb{R}_{0} \times \mathbb{R} \rightarrow$ $\mathbb{R}$ has continuous partial derivatives of the second-order and $\partial^{2} f / \partial x \partial y \in L_{1}\left(\left[0, b^{*} / m\right] \times[c, d]\right)$ with $0 \leq a<b \leq b^{*}$, $c<d$, and $0 \leq r \leq q,-1<\ell \leq q$. If $\left|\partial^{2} f / \partial x \partial y\right|^{q}$ is coordinated $(s, m)$-P-convex functions on $\left[0, b^{*} / m\right] \times[c, d]$ for some $m \in(0,1], s \in(-1,1]$, and $q>1$, then

$$
\begin{aligned}
& |P(a, b, c, d)| \leq \frac{(b-a)(d-c)}{\left[2^{\ell}(\ell+1)\right]^{1 / q}}\left(\frac{(q-1)^{2}}{(2 q-r-1)(2 q-\ell-1)}\left(\frac{1}{2}\right)^{(4 q-r-\ell-2) /(q-1)}\right)^{1-1 / q} \\
& \quad \times\left\{\left[\frac{\Delta_{1}(q)}{2^{r+s+1}(r+s+1)}+\frac{2^{-s} m \Delta_{2}(m, q)}{r+1}{ }_{2} F_{1}\left(-s, r+1, r+2,2^{-1}\right)\right]^{1 / q}\right. \\
& \left.\quad+\left[\left[B(r+1, s+1)-2^{-s} F_{1}\left(-r, s+1, s+2,2^{-1}\right)\right] \frac{\Delta_{1}(q)}{s+1}+\frac{m \Delta_{2}(m, q)}{2^{r+s+1}(r+s+1)}\right]^{1 / q}\right\}
\end{aligned}
$$

where $\Delta_{1}(q)$ and $\Delta_{2}(m, q)$ are defined as in (16), and $B(\alpha, \beta)$ is the beta function defined by

$$
B(\alpha, \beta)=\int_{0}^{1} t^{\alpha-1}(1-t)^{\beta-1} d t, \quad \alpha, \beta>0,
$$

and ${ }_{2} F_{1}(c, d, e, z)$ is the hypergeometric function defined by

$$
\begin{aligned}
& { }_{2} F_{1}(c, d, e, z) \\
& \quad=\frac{\Gamma(e)}{\Gamma(d) \Gamma(e-d)} \int_{0}^{1} t^{d-1}(1-t)^{e-d-1}(1-z t)^{-c} d t
\end{aligned}
$$

for $e>d>0,|z|<1, c \in \mathbb{R}, u>0$.

Proof. Using Lemma 11 and Hölder's integral inequality, we obtain

$$
\begin{aligned}
& |P(a, b, c, d)| \leq(b-a)(d-c)\left\{\left(\int_{0}^{1 / 2} \int_{0}^{1 / 2} t^{(q-r) /(q-1)} \lambda^{(q-\ell) /(q-1)} d t d \lambda\right)^{1-1 / q}\right. \\
& \times\left[\int_{0}^{1 / 2} \int_{0}^{1 / 2} t^{r} \lambda^{\ell}\left|\frac{\partial^{2}}{\partial x \partial y} f(t a+(1-t) b, \lambda c+(1-\lambda) d)\right|^{q} d t d \lambda\right]^{1 / q} \\
& +\left(\int_{1 / 2}^{1} \int_{0}^{1 / 2} t^{(q-r) /(q-1)}(1-\lambda)^{(q-\ell) /(q-1)} d t d \lambda\right)^{1-1 / q} \\
& \times\left[\int_{1 / 2}^{1} \int_{0}^{1 / 2} t^{r}(1-\lambda)^{\ell}\left|\frac{\partial^{2}}{\partial x \partial y} f(t a+(1-t) b, \lambda c+(1-\lambda) d)\right|^{q} d t d \lambda\right]^{1 / q} \\
& +\left(\int_{0}^{1 / 2} \int_{1 / 2}^{1}(1-t)^{(q-r) /(q-1)} \lambda^{(q-\ell) /(q-1)} d t d \lambda\right)^{1-1 / q} \\
& \times\left[\int_{0}^{1 / 2} \int_{1 / 2}^{1}(1-t)^{r} \lambda^{\ell}\left|\frac{\partial^{2}}{\partial x \partial y} f(t a+(1-t) b, \lambda c+(1-\lambda) d)\right|^{q} d t d \lambda\right]^{1 / q} \\
& +\left(\int_{1 / 2}^{1} \int_{1 / 2}^{1}(1-t)^{(q-r) /(q-1)}(1-\lambda)^{(q-\ell) /(q-1)} d t d \lambda\right)^{1-1 / q} \\
& \left.\times\left[\int_{1 / 2}^{1} \int_{1 / 2}^{1}(1-t)^{r}(1-\lambda)^{\ell}\left|\frac{\partial^{2}}{\partial x \partial y} f(t a+(1-t) b, \lambda c+(1-\lambda) d)\right|^{q} d t d \lambda\right]^{1 / q}\right\}
\end{aligned}
$$


After some calculations, it follows that

$$
\begin{aligned}
& \int_{0}^{1 / 2} \int_{0}^{1 / 2} t^{(q-r) /(q-1)} \lambda^{(q-\ell) /(q-1)} d t d \lambda \\
& \quad=\int_{1 / 2}^{1} \int_{0}^{1 / 2} t^{(q-r) /(q-1)}(1-\lambda)^{(q-\ell) /(q-1)} d t d \lambda \\
& =\int_{0}^{1 / 2} \int_{1 / 2}^{1}(1-t)^{(q-r) /(q-1)} \lambda^{(q-\ell) /(q-1)} d t d \lambda \\
& =\int_{1 / 2}^{1} \int_{1 / 2}^{1}(1-t)^{(q-r) /(q-1)}(1-\lambda)^{(q-\ell) /(q-1)} d t d \lambda \\
& =\frac{(q-1)^{2}}{(2 q-r-1)(2 q-\ell-1)} \times 2^{-(4 q-r-\ell-2) /(q-1)} .
\end{aligned}
$$

From the coordinated $(s, m)-P$-convexity of $\left|\partial^{2} f / \partial x \partial y\right|^{q}$, we deduce that

$$
\begin{aligned}
& \int_{0}^{1 / 2} \int_{0}^{1 / 2} t^{r} \lambda^{\ell} \mid \frac{\partial^{2}}{\partial x \partial y} \\
& \left.\cdot f(t a+(1-t) b, \lambda c+(1-\lambda) d)\right|^{q} d t d \lambda \\
& \leq \frac{2^{-(\ell+1)}}{\ell+1} \int_{0}^{1 / 2} t^{r}\left[t^{s} \Delta_{1}(q)+m(1-t)^{s}\right. \\
& \left.\cdot \Delta_{2}(m, q)\right] d t=\frac{2^{-(\ell+1)}}{\ell+1}\left[\frac{\Delta_{1}(q)}{2^{r+s+1}(r+s+1)}\right. \\
& \left.+\frac{2^{-s} m \Delta_{2}(m, q)}{r+1}{ }_{2} F_{1}\left(-s, r+1, r+2,2^{-1}\right)\right] \\
& \int_{1 / 2}^{1} \int_{0}^{1 / 2} t^{r}(1-\lambda)^{\ell} \mid \frac{\partial^{2}}{\partial x \partial y}
\end{aligned}
$$

$$
\begin{aligned}
& \left.f(t a+(1-t) b, \lambda c+(1-\lambda) d)\right|^{q} d t d \lambda \\
& \leq \frac{2^{-(\ell+1)}}{\ell+1}\left[\frac{\Delta_{1}(q)}{2^{r+s+1}(r+s+1)}+\frac{2^{-s} m \Delta_{2}(m, q)}{r+1}\right. \\
& \left.\cdot{ }_{2} F_{1}\left(-s, r+1, r+2,2^{-1}\right)\right] \text {, } \\
& \int_{0}^{1 / 2} \int_{1 / 2}^{1}(1-t)^{r} \lambda^{\ell} \mid \frac{\partial^{2}}{\partial x \partial y} \\
& \left.f(t a+(1-t) b, \lambda c+(1-\lambda) d)\right|^{q} d t d \lambda \\
& \leq \frac{2^{-(\ell+1)}}{\ell+1}\left[\left[B(r+1, s+1)-2^{-s}{ }_{2} F_{1}(-r, s+1, s\right.\right. \\
& \left.\left.\left.+2,2^{-1}\right)\right] \times \frac{\Delta_{1}(q)}{s+1}+\frac{m \Delta_{2}(m, q)}{2^{r+s+1}(r+s+1)}\right], \\
& \int_{1 / 2}^{1} \int_{1 / 2}^{1}(1-t)^{r}(1-\lambda)^{\ell} \mid \frac{\partial^{2}}{\partial x \partial y} \\
& \left.f(t a+(1-t) b, \lambda c+(1-\lambda) d)\right|^{q} d t d \lambda \\
& \leq \frac{2^{-(\ell+1)}}{\ell+1}\left[\left[B(r+1, s+1)-2_{2}^{-s} F_{1}(-r, s+1, s\right.\right. \\
& \left.\left.\left.+2,2^{-1}\right)\right] \times \frac{\Delta_{1}(q)}{s+1}+\frac{m \Delta_{2}(m, q)}{2^{r+s+1}(r+s+1)}\right] .
\end{aligned}
$$

Applying (31) and inequalities (32) into inequality (30), we get inequality (27). The proof of Theorem 15 is complete.

Corollary 16. Under the assumptions of Theorem 15, if $r=0$, then

$$
\begin{aligned}
|P(a, b, c, d)| \leq & \frac{(b-a)(d-c)}{\left[2^{s+\ell+1}(s+1)(\ell+1)\right]^{1 / q}}\left(\frac{(q-1)^{2}}{(2 q-1)(2 q-\ell-1)}\left(\frac{1}{2}\right)^{(4 q-\ell-2) /(q-1)}\right)^{1-1 / q} \\
& \times\left\{\left[\Delta_{1}(q)+m\left(2^{s+1}-1\right) \Delta_{2}(m, q)\right]^{1 / q}+\left[\left(2^{s+1}-1\right) \Delta_{1}(q)+m \Delta_{2}(m, q)\right]^{1 / q}\right\} .
\end{aligned}
$$

In particular, if $r=\ell=0$, then

$$
\begin{aligned}
& |P(a, b, c, d)| \\
& \leq \frac{(b-a)(d-c)}{\left[2^{s+1}(s+1)\right]^{1 / q}}\left(\frac{q-1}{2 q-1}\left(\frac{1}{2}\right)^{(2 q-1) /(q-1)}\right)^{2(1-1 / q)} \\
& \quad \times\left\{\left[\Delta_{1}(q)+m\left(2^{s+1}-1\right) \Delta_{2}(m, q)\right]^{1 / q}\right. \\
& \left.\quad+\left[\left(2^{s+1}-1\right) \Delta_{1}(q)+m \Delta_{2}(m, q)\right]^{1 / q}\right\}
\end{aligned}
$$

\section{Conflicts of Interest}

The authors declare that they have no conflicts of interest.

\section{Authors' Contributions}

All authors read and approved the final manuscript.

\section{Acknowledgments}

This work was partially supported by the Natural Science Foundation of Fujian Province of China (no. 2016J01023), 
the Foundation of the Research Program of Science and Technology at Universities of Inner Mongolia Autonomous Region of China (no. NJZZ18154), and the Science Research Fund of Inner Mongolia University for Nationalities (nos. NMDGP1713 and NMDYB1748).

\section{References}

[1] F. Chen and S. Wu, "Some Hermite-Hadamard type inequalities for harmonically s -convex functions," The Scientific World Journal, vol. 2014, Article ID 279158, 2014.

[2] S. S. Dragomir, "On some new inequalities of HermiteHadamard type for m-convex functions," Tamkang Journal of Mathematics, vol. 33, no. 1, pp. 45-55, 2002.

[3] S. S. Dragomir and S. Fitzpatrick, "The Hadamard inequalities for s-convex functions in the second sense," Demonstratio Mathematica, vol. 32, no. 4, pp. 687-696, 1999.

[4] S. S. Dragomir, J. Pečarić, and L. E. Persson, "Some inequalities of Hadamard type," Soochow Journal of Mathematics, vol. 21, no. 3, pp. 335-341, 1995.

[5] J. Pecaric', F. Proschan, and Y. L. Tong, Convex functions, partial orderings and statistical applications, Academic Press, New York , NY, USA, 1992.

[6] S.-H. Wu, B. Sroysang, J.-S. Xie, and Y.-M. Chu, "Parametrized inequality of Hermite-Hadamard type for functions whose third derivative absolute values are quasi-convex," SpringerPlus, vol. 4, no. 1, article no. 831, 9 pages, 2015.

[7] B.-Y. Xi, R.-F. Bai, and F. Qi, "Hermite-Hadamard type inequalities for the $(\alpha, \mathrm{m})$-geometrically convex functions," Aequationes Mathematicae, vol. 84, no. 3, pp. 261-269, 2012.

[8] B.-Y. Xi and F. Qi, "Some Hermite-Hadamard type inequalities for differentiable convex functions and applications," Hacettepe Journal of Mathematics and Statistics, vol. 42, no. 3, pp. 243-257, 2013.

[9] B.-Y. Xi and F. Qi, "Hermite-Hadamard type inequalities for geometrically r-convex functions," Studia Scientiarum Mathematicarum Hungarica, vol. 51, no. 4, pp. 530-546, 2014.

[10] Y.-M. Bai, S.-H. Wu, and Y. Wu, "Hermite-Hadamard Type Inequalities Associated with Coordinated ((s,m),QC)-convex functions," Journal of Function Spaces, 8 pages, 2017.

[11] S. R. Mohan and S. K. Neogy, "On invex sets and preinvex functions," Journal of Mathematical Analysis and Applications, vol. 189, no. 3, pp. 901-908, 1995.

[12] Z. Pavić, S. Wu, and V. Novoselac, "Application of functionals in creating inequalities," Journal of Function Spaces, vol. 2016, Article ID 9324804, 2016.

[13] Z. Pavic, S. Wu, and V. Novoselac, "Important inequalities for preinvex functions," Journal of Nonlinear Sciences and Applications. JNSA, vol. 9, no. 6, pp. 3570-3579, 2016.

[14] R. Pint, "Invexity and generalized convexity," Optimization. A Journal of Mathematical Programming and Operations Research, vol. 22, no. 4, pp. 513-525, 1991.

[15] S.-H. Wu, I. A. Baloch, and I. Iscan, "On harmonically (p,h,m)preinvex functions," Journal of Function Spaces, Art. ID 2148529, 9 pages, 2017.

[16] Y. Wu, F. Qi, Z.-L. Pei, and S.-P. Bai, "Hermite-Hadamard type integral inequalities via (s,m)-P-convexity on co-ordinates," Journal of Nonlinear Sciences and Applications. JNSA, vol. 9, no. 3, pp. 876-884, 2016.

[17] G. Toader, "Some generalizations of the convexity," in Proceedings of the Colloquium on Approximation and Optimization
(Cluj-Napoca, 1985), pp. 329-338, University of Cluj-Napoca, Cluj, Romania, 1985.

[18] H. Hudzik and L. Maligranda, "Some remarks on s-convex functions," Aequationes Mathematicae, vol. 48, no. 1, pp. 100111, 1994.

[19] J. Park, "Some Hadamard's type inequalities for co-ordinated $(\mathrm{s}, \mathrm{m})$-convex mappings in the second sense," Far East Journal of Mathematical Sciences (FJMS), vol. 51, no. 2, pp. 205-216, 2011.

[20] B.-Y. Xi and F. Qi, "Inequalities of Hermite-Hadamard type for extended s-convex functions and applications to means," Journal of Nonlinear and Convex Analysis. An International Journal, vol. 16, no. 5, pp. 873-890, 2015.

[21] S. S. Dragomir, "On the Hadamard's inequality for convex functions on the co-ordinates in a rectangle from the plane," Taiwanese Journal of Mathematics, vol. 5, no. 4, pp. 775-788, 2001.

[22] S. S. Dragomir and C. E. M. Pearce, Selected Topics on HermiteHadamard Inequalities and Applications, RGMIA Monographs, Victoria University, 2000.

[23] M. A. Latif and S. S. Dragomir, "On some new inequalities for differentiable coordinated convex functions," Journal of Inequalities and Applications, vol. 2012, article no. 28, 2012. 


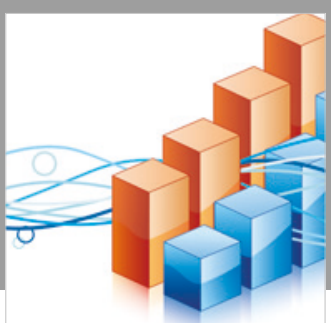

Advances in

Operations Research

\section{-n-m}
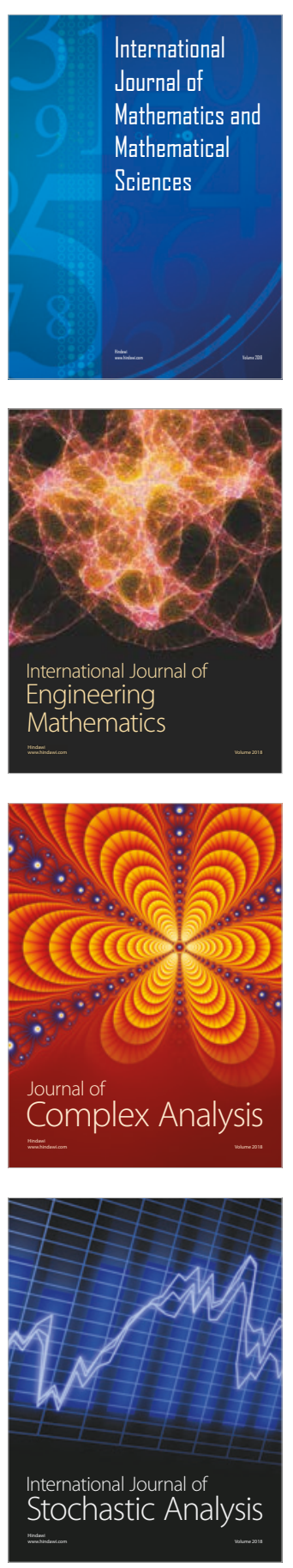
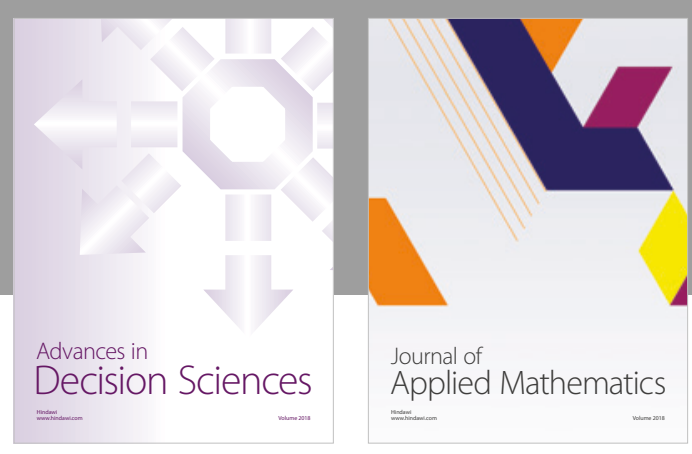

Journal of

Applied Mathematics
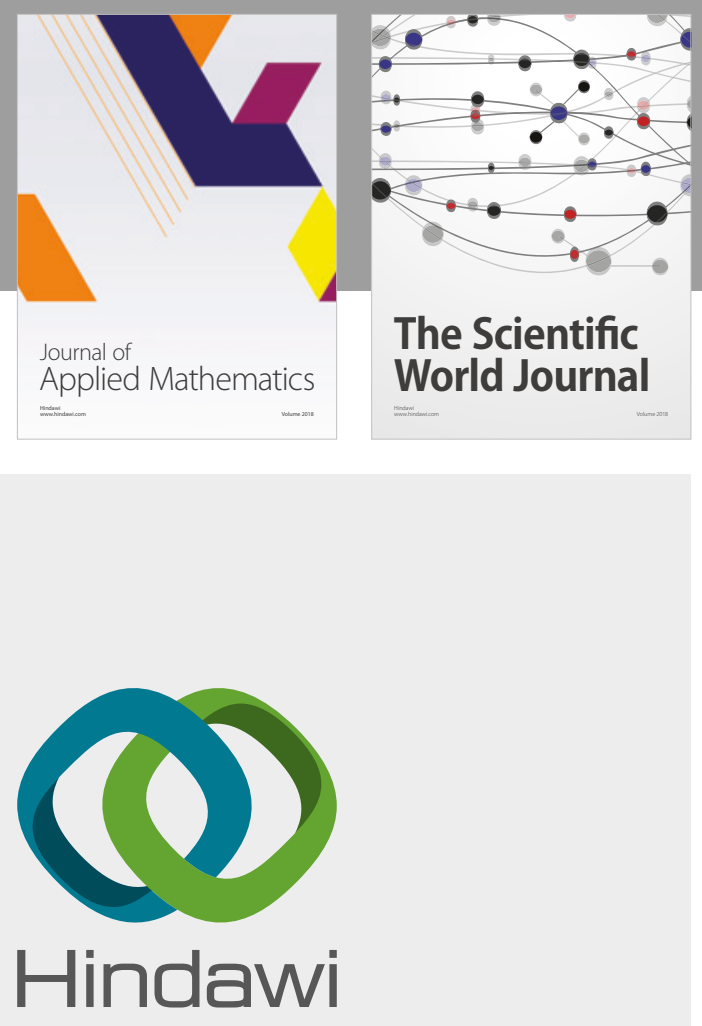

Submit your manuscripts at

www.hindawi.com

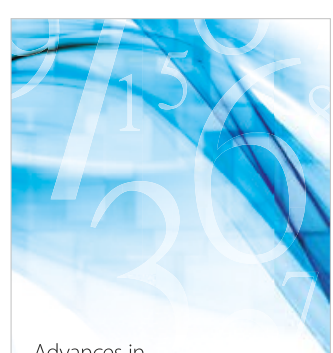

Advances in
Numerical Analysis
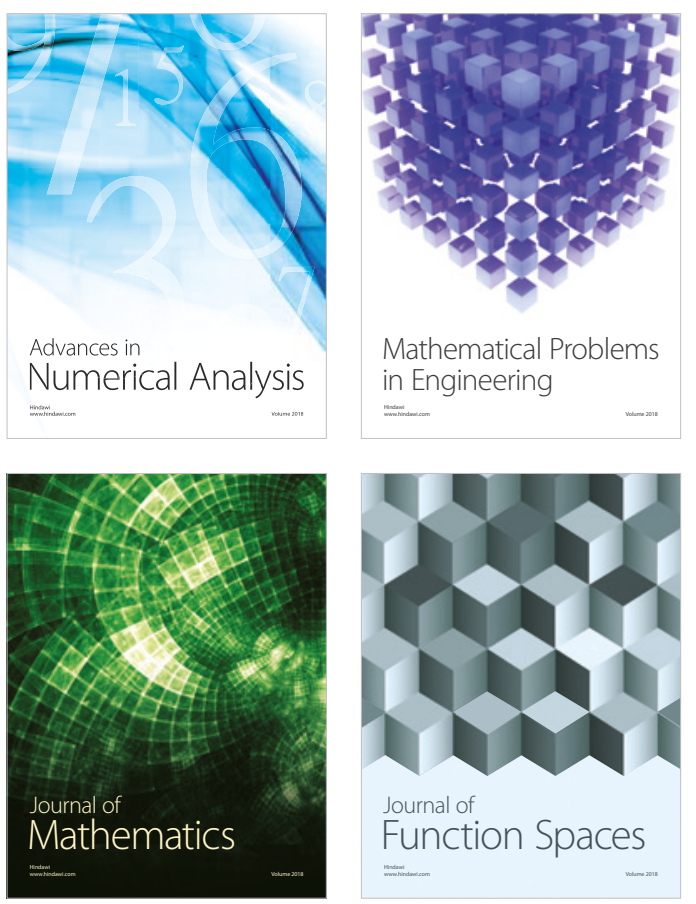

Mathematical Problems in Engineering

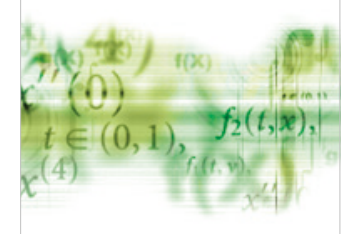

International Journal of

Differential Equations

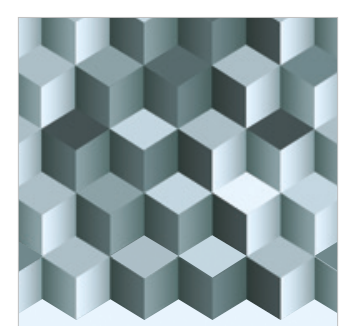

Journal of

Function Spaces

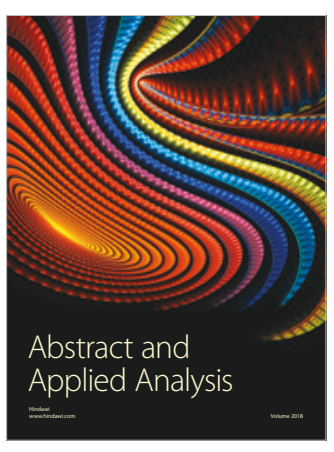

The Scientific

World Journal

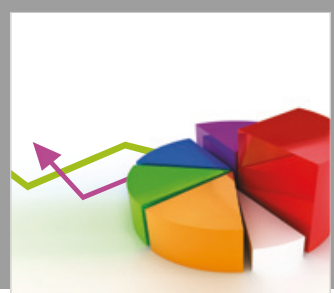

Journal of

Probability and Statistics
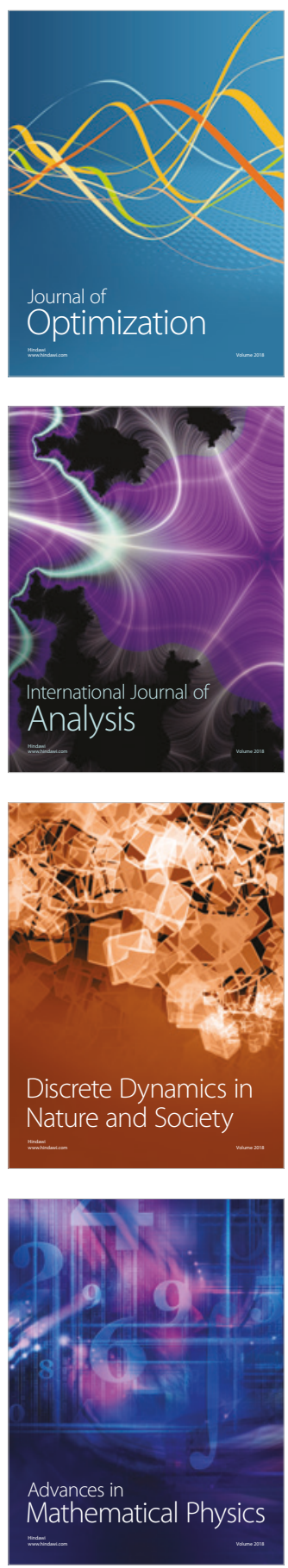